\title{
Randomized Study of Pregabalin in Patients with Cancer-Induced Bone Pain
}

Karl-Fredrik Sjölund • Ruoyong Yang •

Kyung-Hee Lee $\cdot$ Malca Resnick

To view enhanced content go to www.paintherapy-open.com

Received: December 12, 2012 / Published online: February 26, 2013

(c) The Author(s) 2013. This article is published with open access at Springerlink.com

\section{ABSTRACT}

Introduction: Improvements are needed in the management of cancer-induced bone pain (CIBP). The objective of this study was to assess the efficacy and safety of pregabalin compared with placebo in the adjunctive treatment of patients with moderate to severe CIBP who were receiving opioids.

THIS trial is registered at ClinicalTrials.gov, identifier NCT00381095.

K.-F. Sjölund ( $\square)$

ANOPIVA-kliniken, Karolinska University Hospital,

17176 Solna, Sweden

e-mail: karl-fredrik.sjolund@karolinska.se

R. Yang · M. Resnick

Pfizer Inc, New York, NY, USA

K.-H. Lee

Department of Internal Medicine, Yeungnam

University Medical Center, Taegu, Republic of Korea

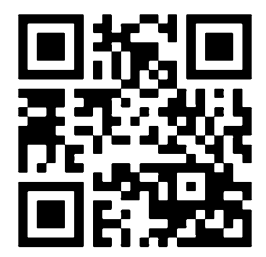

Enhanced content for this article is

available on the journal web site:

www.paintherapy-open.com
Methods: In this randomized, double-blind, placebo-controlled, parallel-group, multicenter trial, 152 adults diagnosed with a malignant, solid tumor with metastases to bone were randomized to flexible-dose pregabalin (100, 150,300 , or $600 \mathrm{mg} /$ day) or placebo, as add-on to stable opioid analgesic therapy, which was optimized prior to the start of the study. The primary efficacy endpoint was the durationadjusted average change (DAAC) from baseline in the daily worst pain at the reference site (measured by 11-point numeric rating scale [NRS]) during the fixed-dosage phase. The study was terminated early following an interim analysis that indicated an increase in sample size would be needed to satisfy statistical assumptions for the primary endpoint. Given the early termination of the study, only descriptive analyses were performed.

Results: The mean (standard deviation) DAAC from baseline in NRS score for the primary endpoint favored pregabalin treatment: -1.53 (1.81) in the pregabalin group and -1.23 (1.74) in the placebo group. Mean DAAC for average pain and sleep interference (NRS) also favored pregabalin. More patients treated with pregabalin reported improvement ("very much 
improved," "much improved," or "minimally improved") based on Patient Global Impression of Change: $81.4 \%$ compared with $70.0 \%$ in the placebo group.

Conclusion: Data from this study indicate that pregabalin use may reduce metastatic bone pain. Due to the incomplete analysis, further study of pregabalin in the management of CIBP is required.

Keywords: Cancer pain; Cancer-induced bone pain; Metastases; Opioid; Pregabalin

\section{INTRODUCTION}

For patients with metastatic disease, cancerinduced bone pain (CIBP) is a common cause of moderate to severe pain [1-3]. The skeleton is the third most common site of metastatic disease [4], with bone involvement reported in $60-84 \%$ of patients with metastases [1]. The presence of bone metastases is predictive of disease progression, and pain typically increases in intensity as the disease evolves. Patients with CIBP experience varying degrees of nociceptive and neuropathic pain [5]. The underlying mechanism of CIBP is complex and can involve a variety of mechanisms, including tumor-derived inflammation, injury or infiltration of sensory neurons in the bone marrow, nerve entrapment, and an imbalance of bone turnover [6-12].

Current management of CIBP is not optimal, with patients often failing to receive adequate pain control. The cause of CIBP pain is multifactorial and thus a multifaceted treatment approach is warranted. Treatment guidelines address this complexity and recommend multiple and concurrent classes of medications as treatment options, recognizing that often the pain does not respond to a single class of medications [13]. Traditional approaches to CIBP management have relied upon the use of nonsteroidal anti-inflammatory agents (NSAIDs), opioids, and radiotherapy [3, 10, 13, 14].

Pain, especially neuropathic pain, which is ongoing and undertreated, is associated with possible adverse outcomes. Psychological distress, physical dysfunction and disability, and poor health-related quality of life escalate in the presence of neuropathic pain $[15,16]$. Without adequate pain relief, a cycle of increased pain leads to further deterioration of the patient's condition. Because the prevalence of neuropathic pain in patients with cancer has been estimated at 19-39\% [17], recommendations for the management of cancer-related pain should specifically address neuropathic pain [13]. Because neuropathic pain may be only partially responsive to opioids, other drug classes must be considered (e.g., tricyclic antidepressants and anticonvulsants) $[13,18]$.

The objective of this study was to assess the efficacy and safety of flexibly dosed pregabalin, an $\alpha 2 \delta$-ligand with analgesic, anticonvulsant, and anxiolytic activity, compared with placebo as add-on therapy to standard-of-care opioids for moderate to severe pain due to bone metastases.

\section{MATERIALS AND METHODS}

\section{Study Design}

This was a randomized, double-blind, flexibledose, placebo-controlled, parallel-group, multicenter trial conducted at 67 centers in 20 countries (across Europe, North America, Latin America, Middle East, and Asia) in patients with CIBP. The study was initiated on December 20, 2006, and completion of the last patient occurred on October 12, 2010. The trial 


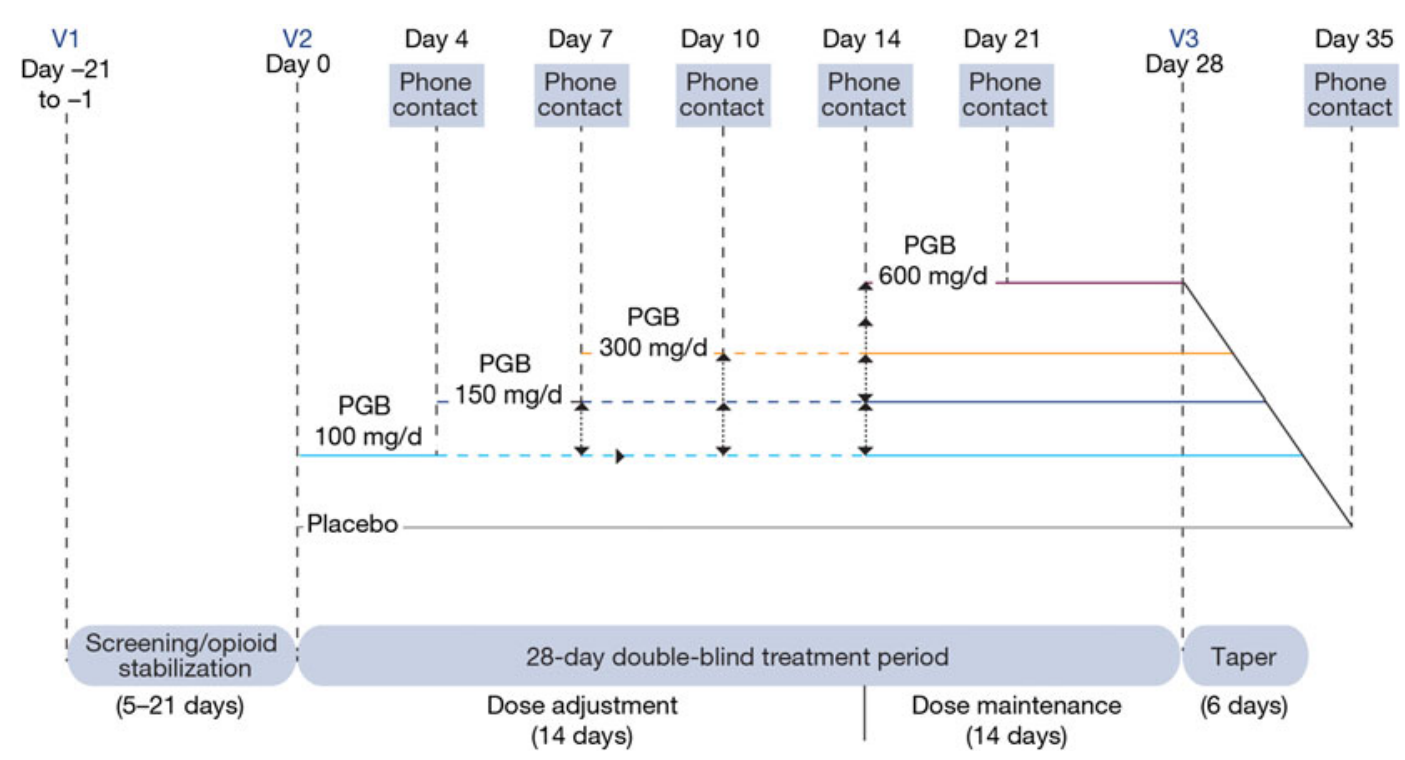

Fig. 1 Study design. $P G B$ pregabalin, $V$ visit

comprised three phases as outlined in Fig. 1: a screening/opioid dose stabilization phase (5-21 days; visit 1 to visit 2 [day 0]); a 28-day, double-blind treatment phase (visit 2 [day 0] to visit 3 [day 28]), consisting of a treatment drug dose-adjustment phase (up to 14 days) and a dose-maintenance phase (lasting until day 28); and a double-blind taper phase (6 days).

\section{Screening/Opioid Dose Stabilization Phase}

Visit 1 included eligibility confirmation and the initiation of opioid stabilization (if needed). Adjustments to the total daily dose of opioids and choice of opioids were permitted during this period until the maximum pain control had been achieved with tolerable side effects; dosage adjustments were allowed for up to 21 days. Stable opioid dose was defined as taking a constant, well-tolerated dose of opioids with no more than four dosages of immediate-release (IR) opioid rescue per day. Once the opioid dose was stable for 5 days, patients with continuing pain who met all eligibility criteria (see below) were randomized into the double-blind treatment phase (visit 2).

\section{Double-Blind Treatment Phase}

Patients eligible for entry into the double-blind treatment phase were randomized to either pregabalin or matching placebo and entered a dose-titration period that lasted up to day 14 . Pregabalin was taken orally, twice daily, upon wakening and at bedtime. Dosages began at $100 \mathrm{mg} /$ day with a goal of reaching a minimum dose of $150 \mathrm{mg}$ /day and a maximum of 600 $\mathrm{mg} /$ day. Dosages were adjusted as outlined in Fig. 1, with dose titration allowed until day 14 . The achieved dosage was then maintained until day 28. A 6-day double-blind taper followed the 28-day double-blind treatment period. During the treatment phase, maintenance of opioid therapy was required; patients could have IR dosages of rescue opioids as needed.

The study was conducted in compliance with the ethical principles originating in or derived from the Declaration of Helsinki. The protocol 
adhered to the International Conference on Harmonization Good Clinical Practice Guidelines. Patients provided written informed consent prior to participation in the trial. The protocol and informed consent documents were reviewed and approved by Institutional Review Boards at participating sites.

\section{Participants}

Patients were aged at least 18 years, had malignant, solid tumors that were diagnosed as having metastasized to bone (with radiographic or scintigraphic confirmation of the site of bone metastases), were able to identify a reference site for pain with a score of $\geq 4$ on an 11-point numeric rating scale (NRS) at the reference site at screening (visit 1), and had a life expectancy of $\geq 3$ months from the start of the study were eligible for inclusion. The reference site of pain was judged clearly related to known radiographically verified metastases to bone. The possible nociceptive and neuropathic components of the pain at this site were not specifically assessed.

Stable and optimized opioid treatment was an entry criterion for the study. For ethical reasons, and to avoid interference with other treatments used in this population in clinical practice, many stable concomitant medications (skeletal muscle relaxants, antidepressants, benzodiazepines, and NSAIDS) were allowed to continue. Medications not permitted during the study period included antiepileptics, barbiturates, monoamine oxidase inhibitors, retinotoxins, and N-methyl-D-aspartate (NMDA) receptor antagonists. Chemotherapy and radiotherapy/radiopharmaceutical treatments were continued if the patient was stable with use and there being no plan to initiate or change therapy during the course of the study. In patients who were receiving radiotherapy, treatment had to have been initiated $\geq 15$ days prior to study screening.

For entry into the double-blind treatment phase, an average score of $\geq 4$ for the daily worst pain at the reference site during the week prior to randomization was required. Patients were receiving a stable daily dose of opioid (established during the opioid optimization phase prior to randomization), and were allowed to receive no more than four dosages of IR opioid rescue medication per day in the week preceding randomization. Patients with other clinically significant disease or with any current psychiatric disorder were excluded from the study. Patients were also excluded if they had mechanical or radicular back pain or had undergone invasive interventions in the 15 days prior to the study.

\section{Study Evaluations}

\section{Daily Numeric Rating Scale}

Pain was reported on the NRS scale of $0-10$, with 0 being "no pain" and 10 being "pain as bad as can be imagined." Sleep interference was reported on a similar scale, with 0 equals "pain has no interference with sleep" and 10 equals "pain completely interferes with sleep." The 11-point NRS scores were collected (via paper diary) each evening before bedtime for the assessment of daily average pain, worst pain overall, and pain at reference site and each morning for pain-related sleep interference.

\section{Modified Brief Pain Inventory Short Form}

The Brief Pain Inventory (BPI) is a tool for assessing chronic pain originally developed for use in patients with cancer [19-21]. This tool measures the intensity of pain based on an 11 -point response scale (0-10) and is considered a useful outcome measure for patients with advanced cancer. The modified BPI Short Form 
(mBPI-sf) was completed by patients at baseline and at the end of treatment.

\section{Hospital Anxiety and Depression Scale}

The Hospital Anxiety and Depression Scale (HADS) is a 14-item questionnaire with two subscales: one measures anxiety (HADS-A) and the other measures depression (HADS-D) [22]. Each subscale (scale of $0-3$ ) consists of seven statements to which patients respond indicating how well the item applies to them. Total scores range from 0 to 21 , with higher scores indicating a greater severity of symptoms. Anxiety and depression using HADS were reported by patients at baseline and at the end of treatment.

\section{Patient Global Impression of Change}

The Patient Global Impression of Change (PGIC) is a patient-rated instrument that measures change in a patient's overall status on a 7-point scale ranging from 1 (very much improved) to 7 (very much worse). Statistically significant pain score reductions have been verified as being clinically meaningful using PGIC [23]. Scores were reported by patients at the end of the fixed-dosing period (week 4) and at the end of treatment.

\section{Primary and Secondary Efficacy Endpoints}

The primary efficacy endpoint was durationadjusted average change (DAAC) from baseline in the daily NRS worst pain at the reference site during the fixed-dosage double-blind phase (first day on stable dose through to day 28). This was defined as the area under the curve of change in worst pain after reaching a stable dose of placebo or pregabalin divided by the treatment duration of the fixed-dosage period. Secondary efficacy endpoints included: (1) DAAC during the fixed-dosage period for NRS average pain and NRS sleep interference scores; (2) change in mBPI-sf pain severity index, mBPIsf pain interference index, worst pain score, average pain score, and sleep interference score; (3) HADS change from baseline by visit and last observation carried forward (LOCF) for anxiety and depression; and (4) change in PGIC score from baseline.

In addition, responder rates were considered on two levels: $30 \%$ responder (percent change from baseline in NRS worst pain at reference site score $\geq 30 \%$ ) and $50 \%$ responder (percent change from baseline in NRS worst pain at the reference site score $\geq 50 \%$ ).

\section{Safety Assessments}

Safety and tolerability were assessed by monitoring adverse events (AEs), including clinically significant symptoms and signs, abnormal laboratory test values, and changes in physical examination findings. Weight, supine and standing blood pressure, and pulse were assessed at all scheduled visits. Laboratory assessments, physical examination, and neurologic examination were completed at screening and endpoint and a 12-lead electrocardiogram was obtained at visit 1 . The AEs were collected at all visits and telephone contacts.

\section{Statistical Analysis}

SAS v.8.2 (SAS Institute, Cary, NC, www.sas.com) were used for generating all statistical summary tables. A pre-planned sample size of 108 participants per group (216 in total) was estimated to achieve $90 \%$ power with the detection of a 0.8-point difference between treatment groups in the DAAC of worst pain. The intent-to-treat population was used for the primary analyses and included all randomized 
patients who received at least one dose of double-blind medication and had at least one postbaseline efficacy evaluation. The LOCF endpoint was defined as the last, post-baseline observation carried forward. Two pre-specified interim analyses were performed. A blinded interim analysis occurred when 80 randomized patients had either completed or withdrawn early from the study. An unblinded interim analysis was performed after $50 \%(n=108)$ of patients had completed/withdrawn for sample size adjustment. Based on the second interim analysis, it was determined that an increase in sample size would be needed to satisfy statistical assumptions.

The study was terminated early because the increase in sample size, combined with slow enrollment, would require a significant extension to the study duration. With the early termination of the study, only descriptive analyses were performed.

\section{RESULTS}

\section{Patient Population}

A total of 152 patients were enrolled in the study; 72 patients were randomized to pregabalin and 80 to placebo (Fig. 2). Eightytwo percent of patients in the pregabalin group and $74 \%$ in the placebo group completed the study. Baseline characteristics were similar between treatment groups (Table 1). The most common types of cancer represented were breast (pregabalin 29.2\%, placebo 30.0\%), prostate $(22.2 \%$ pregabalin, $27.5 \%$ placebo), lung $(9.7 \%$ pregabalin, $16.3 \%$ placebo), and kidney $(6.9 \%$ pregabalin, $10.0 \%$ placebo). Mean duration since the diagnosis of metastatic pain was 0.9 years in the pregabalin group and 1.4 years in the placebo group. The mean duration of study treatment was 30 days

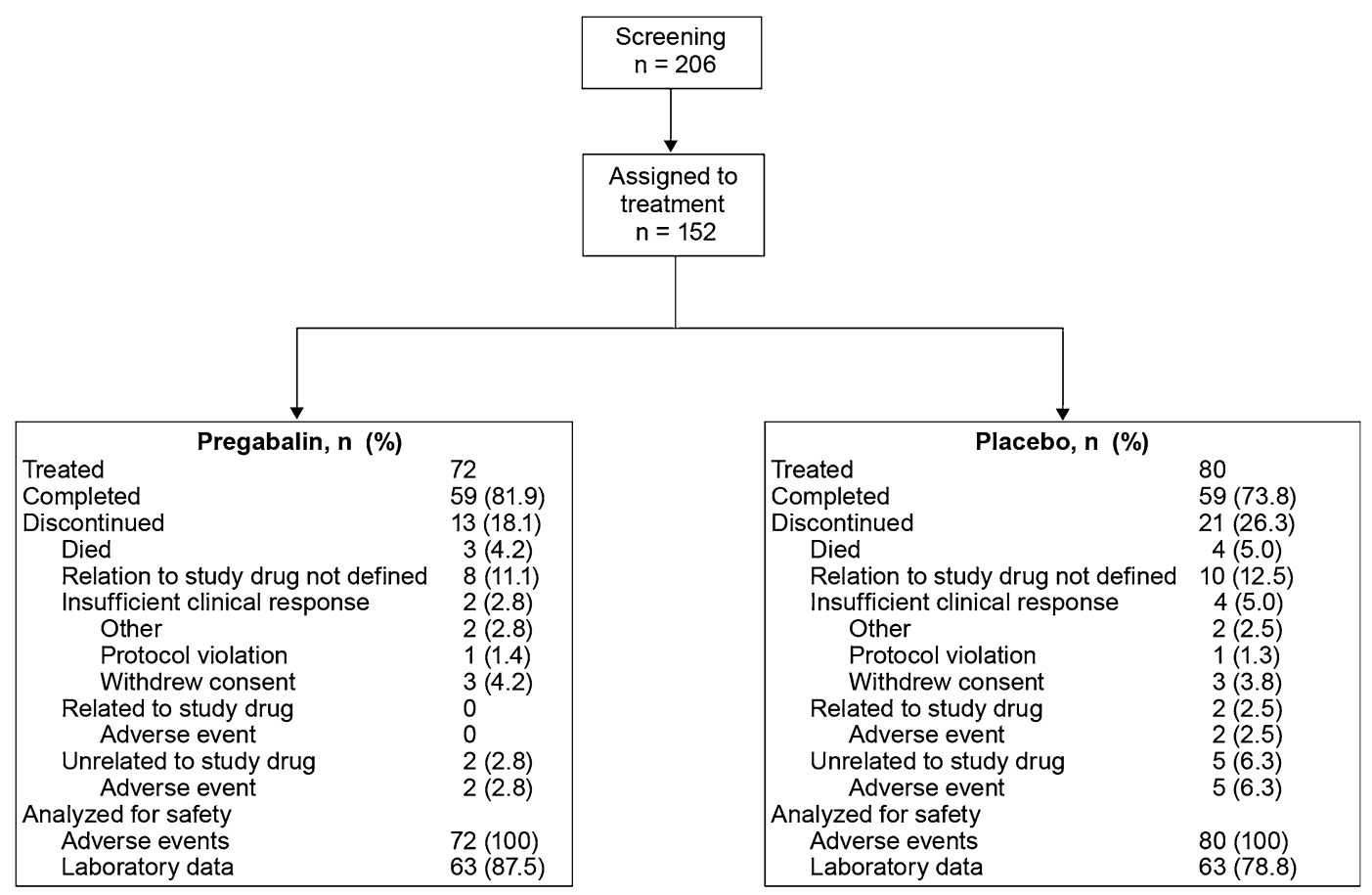

Fig. 2 Patient disposition 
Table 1 Patient demographics

\begin{tabular}{|c|c|c|}
\hline & $\begin{array}{l}\text { Pregabalin } \\
(n=72)\end{array}$ & $\begin{array}{l}\text { Placebo } \\
(n=80)\end{array}$ \\
\hline \multicolumn{3}{|l|}{ Gender, $n(\%)$} \\
\hline Male & $36(50.0)$ & $39(48.8)$ \\
\hline Female & $36(50.0)$ & $41(51.3)$ \\
\hline Mean age $\pm S D$, years & $58.2 \pm 11.3$ & $59.9 \pm 11.9$ \\
\hline \multicolumn{3}{|l|}{ Age, years, $n(\%)$} \\
\hline $18-44$ & $7(9.7)$ & $7(8.8)$ \\
\hline $45-64$ & $43(59.7)$ & $47(58.8)$ \\
\hline$\geq 65$ & $22(30.6)$ & $26(32.5)$ \\
\hline \multicolumn{3}{|l|}{ Race, $n(\%)$} \\
\hline White & $44(61.1)$ & $47(58.8)$ \\
\hline Black & $2(2.8)$ & $2(2.5)$ \\
\hline Asian & $17(23.6)$ & $19(23.8)$ \\
\hline Other & $9(12.5)$ & $12(15.0)$ \\
\hline Mean weight $\pm S D, \mathrm{~kg}$ & $67.1 \pm 16.4$ & $68.1 \pm 17.5$ \\
\hline Mean height $\pm S D, c m$ & $165.4 \pm 10.9$ & $163.3 \pm 9.9$ \\
\hline $\begin{array}{l}\text { Mean duration since } \\
\text { diagnosis of metastatic } \\
\text { pain, years (range) }\end{array}$ & $0.9(0-5.4)$ & $1.4(0-16.3)$ \\
\hline
\end{tabular}

for pregabalin and 35 days for placebo. Other than opioids, the most common concomitant medications received were related to chemotherapy and the management of symptoms related to chemotherapy regimens. Two patients in the placebo group and no patients in the pregabalin group had radiotherapy during the study period.

\section{Primary Endpoint}

\section{NRS Worst Pain}

The mean (standard deviation [SD]) DAAC from baseline in NRS score for the primary endpoint of worst pain at the reference site (fixed-dosage
Table 2 Summary of duration-adjusted average change (DAAC) scores during fixed-dosage period

\begin{tabular}{|c|c|c|}
\hline & \multicolumn{2}{|c|}{ Mean change (SD) } \\
\hline & Pregabalin & Placebo \\
\hline NRS worst pain ${ }^{\mathrm{a}}$ & $-1.53(1.81)$ & $-1.23(1.74)$ \\
\hline NRS average pain ${ }^{a}$ & $-1.24(1.65)$ & $-0.85(1.59)$ \\
\hline NRS sleep interference ${ }^{b}$ & $-1.37(2.02)$ & $-0.63(1.78)$ \\
\hline
\end{tabular}

DAAC computed by area under the curve of each pain score by trapezoidal's rule, then divided by days in each period

$N R S$ numeric rating scale, $S D$ standard deviation

a Scale of $0-10$, with 0 being "no pain" and 10 being "pain as bad as you can imagine"

b Scale of $0-10$, with 0 being "does not interfere" and 10 being "completely interferes"

period) favored pregabalin: -1.53 (1.81) in the pregabalin group and -1.23 (1.74) in the placebo group (Table 2).

\section{Secondary Endpoints}

\section{NRS Average Pain}

The mean (SD) DAAC from baseline in NRS average pain score during the fixed-dosing period favored pregabalin treatment: a reduction in pain of -1.24 (1.65) in the pregabalin group and -0.85 (1.59) in the placebo group (Table 2).

\section{NRS Sleep Interference}

The mean (SD) DAAC from baseline in NRS sleep interference score also favored pregabalin, showing a reduction in sleep interference: -1.37 (2.02) for the pregabalin group and -0.63 (1.78) in the placebo group (Table 2).

\section{mBPI-sf Scores}

Pregabalin demonstrated numerically greater mean decreases (improvement) in all of the mBPI-sf measures (pain severity index, pain 
Table 3 Mean change in mBPI-sf scores

\begin{tabular}{lll}
\hline & \multicolumn{2}{l}{ Mean change (SD) } \\
\cline { 2 - 3 } & Pregabalin & Placebo \\
\hline Pain severity index & $-1.94(1.88)$ & $-1.35(2.54)$ \\
Pain interference index & $-1.66(2.57)$ & $-1.48(2.56)$ \\
Worst pain score & $-2.31(2.48)$ & $-1.63(2.77)$ \\
Average pain score & $-2.09(2.01)$ & $-1.35(2.45)$ \\
Sleep interference & $-2.08(2.73)$ & $-1.47(3.05)$ \\
\hline
\end{tabular}

Baseline to last observation carried forward

$m B P I-s f$ modified Brief Symptom Inventory Short Form, $S D$ standard deviation

interference index, worst pain score, average pain score, and sleep interference) when compared with placebo (Table 3).

\section{HADS}

HADS-A and HADS-D subscale scores showed numerically greater increases (indicating greater severity) in anxiety and depression scores with pregabalin treatment when compared with placebo. The mean (SD) change in anxiety score from baseline to endpoint (LOCF) with pregabalin was 0.80 (3.37) compared with 0.71 (2.42) with placebo. The mean (SD) change in depression score from baseline to LOCF with pregabalin was 0.22 (2.73) compared with -0.57 (2.81) with placebo.

\section{PGIC Scores}

The mean (SD) PGIC scores at the end of the fixeddosing period (week 4) and LOCF showed lower mean scores (indicating greater improvement) with pregabalin when compared with placebo, respectively: at week $4,2.73$ (1.39) versus 2.87 (1.46) and at endpoint, 2.88 (1.46) versus 2.99 (1.45). At week 4, 81.4\% of patients treated with pregabalin reported improvement in PGIC ("very much improved," "much improved," or

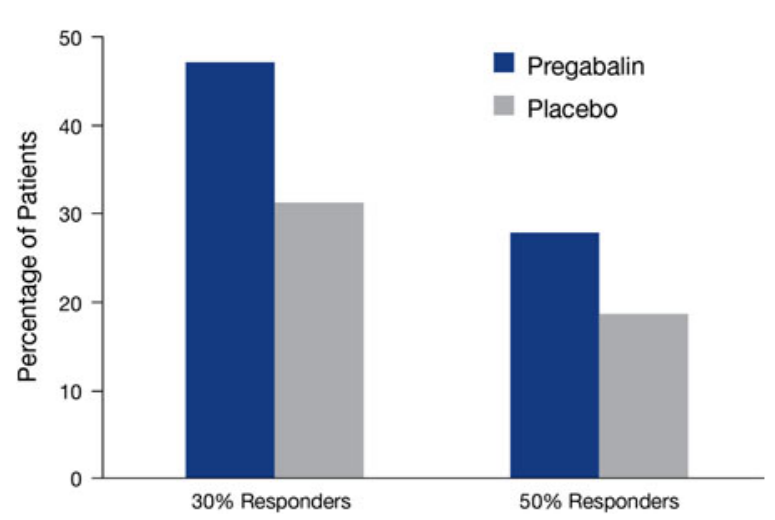

Fig. 3 Responders by treatment. A 30\% responder = patient with percent change from baseline in numeric rating scale (NRS) worst pain at reference site score of $\geq 30 \%$; a $50 \%$ responder $=$ patient with percent change from baseline in NRS worst pain at reference site score of $\geq 50 \%$. Treatment is at last observation carried forward

"minimally improved") compared with $70.0 \%$ of patients treated with placebo.

\section{Responder Rates}

More patients treated with pregabalin reported a $30 \%$ or $50 \%$ reduction in pain compared with those treated with placebo at LOCF. Figure 3 shows the patients achieving a $30 \%$ or $50 \%$ reduction in NRS worst pain from baseline to LOCF in both treatment groups. Differences in pain reduction between treatment groups were evident by week 1 .

\section{Safety}

A total of 14 deaths $(n=6$, pregabalin; $n=8$, placebo) were reported during the study; 24 serious AEs (SAEs) were reported $(n=12$ in each group). None of the deaths or SAEs occurring during the study period was considered related to study medication in the opinion of the study investigator. Discontinuations due to AEs were experienced by $4.2 \%$ of patients in the pregabalin group and $13.8 \%$ of patients in the placebo group. None of the discontinuations in the pregabalin group were considered related to 
study medication. Two (2.5\%) patients in the placebo group discontinued because of AEs related to the study medication, including tachycardia, chest discomfort, and fatigue in the first patient and tachycardia, vomiting, and coughing in the second patient. Other AEs in patients treated with placebo that led to discontinuation (not treatment-related) included disease progression, thrombocytopenia, femur fracture, cardiac arrest, metastases, septic shock, performance status decrease, nausea, and bone pain. The AEs reported in patients treated with pregabalin that led to discontinuation (not treatment-related) included renal failure, disease progression, and performance status decrease.

The most common treatment-emergent AEs (TEAEs) regardless of causality and occurring in $\geq 5 \%$ of patients are reported in Table 4 . Dosage reduction for AEs occurred in $9.7 \%$ of pregabalin-treated patients and $6.3 \%$ of patients receiving placebo.

\section{DISCUSSION}

While only descriptive data are available, DAAC for NRS worst pain at the reference site, average pain, and sleep interference favored pregabalin use in adult patients with CIBP. A greater proportion of patients treated with pregabalin also achieved a $30 \%$ or $50 \%$ reduction in NRS worst pain at the reference site. The effect size in primary outcome measures was small, on a group level smaller than what could be considered clinically important. The higher number in the active treatment group reaching $30 \%$ or $50 \%$ NRS worst pain reduction may, however, indicate still clinically important effect in some patients.

Patients treated with pregabalin had higher scores for anxiety and depression than those receiving placebo; however, the authors do not believe these differences to be clinically
Table 4 Treatment-emergent adverse events ( $\geq 5 \%$, all causalities)

\begin{tabular}{lll}
\hline & \multicolumn{1}{l}{$\boldsymbol{n ( \% )}$} \\
\cline { 2 - 3 } & $\begin{array}{l}\text { Pregabalin } \\
(\boldsymbol{n}=\mathbf{7 2})\end{array}$ & $\begin{array}{l}\text { Placebo } \\
(\boldsymbol{n}=\mathbf{8 0})\end{array}$ \\
\hline Somnolence & $18(25.0)$ & $6(7.5)$ \\
Dizziness & $11(15.3)$ & $7(8.8)$ \\
Nausea & $7(9.7)$ & $10(12.5)$ \\
Fatigue & $8(11.1)$ & $4(5.0)$ \\
Dyspnea & $7(9.7)$ & $3(3.8)$ \\
Vomiting & $6(8.3)$ & $4(5.0)$ \\
Diarrhea & $5(6.9)$ & $3(3.8)$ \\
Disease progression & $4(5.6)$ & $3(3.8)$ \\
Peripheral edema & $4(5.6)$ & $3(3.8)$ \\
Tremor & $5(6.9)$ & $2(2.5)$ \\
Arthralgia & $4(5.6)$ & $2(2.5)$ \\
Headache & $4(5.6)$ & $2(2.5)$ \\
Cough & $1(1.4)$ & $4(5.0)$ \\
Bone pain & 0 & $4(5.0)$ \\
Disturbance & $4(5.6)$ & 0 \\
in attention & & $4(5.0)$ \\
Dysuria & 0 & 0 \\
Vertigo & $4(5.6)$ &
\end{tabular}

meaningful. This finding could also suggest that the reduction in pain experienced by patients treated with pregabalin is not biased by less anxiety in these patients and is more likely related to a treatment effect.

A greater number of AEs occurred in patients treated with placebo versus pregabalin. Although the study was not completed and reasons for this difference were not investigated, this difference may be related to the greater number of individuals in the oldest age group and the longer duration of metastatic pain in the placebo group. The most frequently reported 
AEs, regardless of cause, were somnolence, dizziness, fatigue, and nausea-all of which occurred more frequently with pregabalin versus placebo. These AEs are consistent with those reported in previous pregabalin clinical trials [24]. The relatively high number of deaths during the study period is not surprising (pregabalin, $n=6$ [8.3\%]; placebo, $n=8$ [10\%]), due to the uncertainly of disease course, despite the study requirement for life expectancy of at least 3 months. However, the authors have found no data on actual survival of patients from the point when opioids are needed for the treatment of metastatic bone pain.

Evaluating pregabalin in this patient population is important because, although treatment guidelines recommend its use in the management of patients with cancer-induced pain [13], pregabalin has not been well studied in this population and product labeling does not cover this indication. Only a few published studies address pregabalin use in cancer-related pain and do not specifically target CIBP [25-29] and, with the exception of two trials $[26,28]$, these were open-label or observational studies. The results of one of the randomized controlled trials [26] suggested a morphine-sparing effect and improvement in neuropathic pain with pregabalin, whereas the other study suggested no analgesic benefit with the addition of pregabalin to morphine therapy [28]. Further well-conducted studies in this population would enable healthcare providers to make fully informed decisions regarding treatment.

Slow enrollment in the current study led to early study termination, and therefore limits the interpretation of the results. Although the reasons for poor enrollment have not been fully investigated, enrollment may have been hampered by patient eligibility considerations as well as patient hesitation to participate in a placebo-controlled study at this stage of disease, especially given the availability of treatments in clinical practice. The study required patients with a stable disease course and no plans to initiate or change chemotherapy/radiotherapy or to use disallowed medications during the course of study, which may also have prevented investigators from enrolling less-stable patients.

In general, it is difficult to enroll cancer patients in clinical trials. According to information from the National Cancer Institute, an estimated $3 \%$ of adult cancer patients participate in clinical trials and $40 \%$ of cancer therapy evaluation trials fail to enroll the minimum pre-planned sample size [30]. However, it is interesting and encouraging to see that a high proportion of patients entering this study completed the protocol. The slow enrollment, possibly due to the above considerations, required adding sites across numerous countries to try to complete enrollment. This led to multiple differences in medical care and varying treatments for this type of cancer and pain, which may have evolved even over the course of this study, and likely contributed to the variability seen in the data and the need for an increased sample size.

In addition to difficulties with enrollment, there are inherent challenges in clinical trial design in this patient population. The current study evaluated pregabalin as an adjuvant therapy for patients with metastatic pain receiving optimal opioid therapy. Although some medications were necessarily restricted, because of the severity of illness of the study population and complexity of care necessary, it is ethically and logistically difficult to restrict all other concomitant medications because they are needed for treatment of the underlying cancer as well as the pain and/or side effects related to therapy. Therefore, use of additional and many varied medications and treatment 
regimens across the enrolling countries may have masked the treatment effect to some extent. Finally, difficulty controlling pain is usually accompanied with a progression in disease; therefore, to combine a relatively stable condition (desirable in a study) with the clinical situation when pain treatment becomes problematic is a challenge.

\section{CONCLUSION}

The limitations and early termination of the study mean that firm conclusions cannot be drawn from the dataset. However, the direction of the data collected in this randomized, double-blind flexible-dosed, placebocontrolled, parallel-group multicenter trial of 152 patients suggests a possibility for improvement in pain when pregabalin is added to optimal opioid therapy. Given the trend toward positive results and the need for improving the management of CIBP, further study of pregabalin in this patient population is warranted.

\section{ACKNOWLEDGMENTS}

This study was funded by Pfizer Inc. Medical writing support was provided by Charlotte Kenreigh, PharmD, of UBC Scientific Solutions, and funded by Pfizer Inc. Portions of the findings reported in this manuscript were presented at the 14th World Congress on Pain; August 27-31, 2012; Milan, Italy. Dr. Sjölund is the guarantor for this article, and takes responsibility for the integrity of the work as a whole.

Conflict of interest. Karl-Fredrik Sjölund and Kyung-Hee Lee were investigators for this study. Ruoyong Yang and Malca Resnick are full-time employees of Pfizer Inc.

Open Access. This article is distributed under the terms of the Creative Commons Attribution Noncommercial License which permits any noncommercial use, distribution, and reproduction in any medium, provided the original author(s) and the source are credited.

\section{REFERENCES}

1. Mercadante S. Malignant bone pain: pathophysiology and treatment. Pain. 1997;69: $1-18$.

2. Portenoy RK, Hagen NA. Breakthrough pain: definition, prevalence and characteristics. Pain. 1990;41:273-81.

3. Delaney A, Fleetwood-Walker SM, Colvin LA, Fallon M. Translational medicine: cancer pain mechanisms and management. Br J Anaesth. 2008;101:87-94.

4. Tubiana-Hulin M. Incidence, prevalence and distribution of bone metastases. Bone. 1991;12: S9-10.

5. Caraceni A, Portenoy RK. An international survey of cancer pain characteristics and syndromes. IASP Task Force on Cancer Pain. International Association for the Study of Pain. Pain. 1999;82: 263-74.

6. Colvin L, Fallon M. Challenges in cancer pain management-bone pain. Eur J Cancer. 2008;44: 1083-90.

7. Donovan-Rodriguez T, Dickenson AH, Urch CE. Gabapentin normalizes spinal neuronal responses that correlate with behavior in a rat model of cancer-induced bone pain. Anesthesiology. 2005;102:132-40.

8. Honore $\mathrm{P}$, Rogers SD, Schwei MJ, et al. Murine models of inflammatory, neuropathic and cancer pain each generates a unique set of neurochemical changes in the spinal cord and sensory neurons. Neuroscience. 2000;98:585-98.

9. Luger NM, Sabino MA, Schwei MJ, et al. Efficacy of systemic morphine suggests a fundamental difference in the mechanisms that generate bone 
cancer vs. inflammatory pain. Pain. 2002;99: 397-406.

10. Middlemiss T, Laird BJ, Fallon MT. Mechanisms of cancer-induced bone pain. Clin Oncol (R Coll Radiol). 2011;23:387-92.

11. Peters CM, Ghilardi JR, Keyser CP, et al. Tumorinduced injury of primary afferent sensory nerve fibers in bone cancer pain. Exp Neurol. 2005;193:85-100.

12. Urch CE, Donovan-Rodriguez T, Dickenson AH. Alterations in dorsal horn neurones in a rat model of cancer-induced bone pain. Pain. 2003;106: 347-56.

13. Ripamonti CI, Bandieri E, Roila F. Management of cancer pain: ESMO clinical practice guidelines. Ann Oncol. 2011;22:vi69-77.

14. Caraceni A, Hanks G, Kaasa S, et al. Use of opioid analgesics in the treatment of cancer pain: evidence-based recommendations from the EAPC. Lancet Oncol. 2012;13:e58-68.

15. Portenoy RK, Payne D, Jacobsen P. Breakthrough pain: characteristics and impact in patients with cancer pain. Pain. 1999;81:129-34.

16. Haanpää M, Attal N, Backonja M, et al. NeuPSIG guidelines on neuropathic pain assessment. Pain. 2011;152:14-27.

17. Bennett MI, Rayment C, Hjermstad M, Aass N, Caraceni A, Kaasa S. Prevalence and aetiology of neuropathic pain in cancer patients: a systematic review. Pain. 2011;153:359-65.

18. Vadalouca A, Raptis E, Moka E, Zis P, Sykioti P, Siafaka I. Pharmacological treatment of neuropathic cancer pain: a comprehensive review of the current literature. Pain Pract. 2012;12: 219-51.

19. Cleeland CS, Ryan KM. Pain assessment: global use of the Brief Pain Inventory. Ann Acad Med Singapore. 1994;23:129-38.

20. Serlin RC, Mendoza TR, Nakamura Y, Edwards KR, Cleeland CS. When is cancer pain mild, moderate or severe? Grading pain severity by its interference with function. Pain. 1995;61:277-84.
21. Twycross R, Harcourt J, Bergl S. A survey of pain in patients with advanced cancer. J Pain Symptom Manage. 1996;12:273-82.

22. Zigmond AS, Snaith RP. The hospital anxiety and depression scale. Acta Psychiatr Scand. 1983;67: 361-70.

23. Farrar JT, Young JP Jr, LaMoreaux L, Werth JL, Poole RM. Clinical importance of changes in chronic pain intensity measured on an 11-point numerical pain rating scale. Pain. 2001;94:149-58.

24. Zaccara G, Gangemi P, Perucca P, Specchio L. The adverse event profile of pregabalin: a systematic review and meta-analysis of randomized controlled trials. Epilepsia. 2011;52:826-36.

25. Mańas A, Ciria JP, Fernández MC, et al. Post hoc analysis of pregabalin vs. non-pregabalin treatment in patients with cancer-related neuropathic pain: better pain relief, sleep and physical health. Clin Transl Oncol. 2011;13:656-63.

26. Mishra S, Bhatnagar S, Goyal GN, Rana SP, Upadhya SP. A comparative efficacy of amitriptyline, gabapentin, and pregabalin in neuropathic cancer pain: a prospective randomized double-blind placebo-controlled study. Am J Hosp Palliat Care. 2012;29:177-82.

27. Vondracek P, Oslejskova H, Kepak T, et al. Efficacy of pregabalin in neuropathic pain in paediatric oncological patients. Eur J Paediatr Neurol. 2009; 13:332-6.

28. Mercadante S, Porzio G, Aielli F, et al. The effects of low doses of pregabalin on morphine analgesia in advanced cancer patients. Clin J Pain. 2013;29: $15-9$.

29. Toelle T, Varvara R, Nimour M, Emir B, Brasser M. Pregabalin in neuropathic pain related to DPN, cancer and back pain: analysis of a 6-week observational study. Open Pain J. 2011;5:1-11.

30. English R, Lebovitz Y, Griffin R, Institute of Medicine (US) Forum on Drug Discovery Development and Translation. Transforming clinical research in the United States. Challenges and opportunities: workshop summary. Washington, DC: National Academies Press; 2010. 